12. Brown, G. C., Berryman, A. A. \& Bogyo, T. P. Envir. Ent. 7, 222 (1978).

3. Menšutkin, V. V., Žakov, L. A. \& Umnov, A. A. Vopr Icht. 8, 881-891 (1968)

14. May, R. M. Stability and Complexity in Mode Ecosystems (Princeton University Press, 1973)

15. Beddington, J. R. \& Lawton, J. H. J. Phys. 39, Colloque

\section{Bombesin—satiety or malaise?}

IT has recently been claimed by Gibbs et al. ${ }^{1}$ that bombesin (BBS), like cholecystokinin (CCK), produces satiety in rats. However, their report ignores crucial evidence and lacks critical controls.

First, there is good evidence that CCK suppresses feeding because it produces malaise. This has been shown by appropriate conditioned taste aversion tests ${ }^{2}$. Reports of aversive symptoms in human subjects injected with small doses of CCK corroborate the evidence from rats $^{3}$. Further, doses of CCK that produce food intake suppression produce abnormal patterns of duodenal activity, quite different from the patterns observed during normally induced satiety, from which it is concluded that the amounts of CCK injected to produce intake suppression are much larger than those that are normally secreted ${ }^{4}$

Second, Gibbs et al. ${ }^{1}$ present no relevant evidence to show that BBS in the dose injected is not an aversive agent. It is well known ${ }^{5.6}$ that even quite powerful doses of some agents producing conditioned taste aversion produce no observable symptoms of distress. Moreover, only a very small dose of one such agent $(\mathrm{LiC1})$ is necessary to suppress food intake to the extent reported for BBS ${ }^{7}$. Gibbs et al. ${ }^{1}$ did not use the conditioned taste aversion test to screen for aversive effects. (In such a test a taste is followed by a dose of the agent being tested for aversive properties. If the taste is avoided in a subsequent test, this shows that the agent is indeed aversive.) It has been argued that the results of such a test are ambiguous: conditioned satiation $^{8}$ could lead to a reduction of intake in the same way as conditioned aversion; or, satiation and mild discomfort or malaise may in fact be identical. Both such arguments are contradicted by the experimental evidence. Conditioned satiation would resemble conditioned aversion in a situation where the rat is presented only with the conditioned taste in the test (the so-called single bottle test). However, in a two bottle test where the rat chooses between a neutral solution and the conditioned solution, experiments have shown that when the proper nutrient solutions 9.10 as well as other reinforcers $^{11}$ are paired with a taste then that taste is preferred over a neutral taste in a subsequent test. On the other hand, when aversive agents are paired with a taste, that taste is avoided ${ }^{12,13}$
Clearly, conditioned satiation leads to the opposite result that conditioned aversion does. Such results also dispose of the second argument. If satiation was aversive, stimuli conditioned to it would not be preferred. Even tastes paired with very weakly aversive stimuli produce an aversion ${ }^{7}$. Without evidence from proper behavioural screening tests, the claim that bombesin is a satiety agent cannot be taken seriously. At present it seems most probable that BBS, like CCK, is being administered in unphysiological doses and is therefore suppressing food intake by producing malaise.

\section{J. A. DeutsCh}

Department of Psychology,

University of California,

San Diego, La Jolla,

California 92093

I. Gibbs, J. et al. Nature 282, 208-210 (1979).

2. Deulsch, J. A. \& Hardy, W. T. Nalure 266, 196 (1977).

3. Sturdevant, R. A. L. \& Goetz, H. Nature 261, 713-715 (1976).

4. Deutsch, J. A. Thiel T. R. \& Greenbers L. H. Behas. Biol. 24, 393-399 (1978).

5. Gamzu, E. in Learning Mechanisms in Food Selection ch 19 (eds Barker, L. M., Best, M. R. \& Domjan, M.) 477-509 (Baylor Univ. Press, 1977).

6. Barker, L. M., Smith, J. C. \& Suarez, E. M. in Learning Mechanisms in Food Selection ch. 21 (eds Barker, L. M., Best, M. R. \& Domjan, M.) 533--553 (Baylor Univ. Press, 1977).

7. Deutsch J. A Gonzalez, M. Beha: Biol. 24, 317-320 (1978)

8. Booth, D. A. J. comp. Physiol. Psychol. 81, 457471 (1972).

9. Holman, G. L. J. cump. Physiol. Psychol. 69, 432-441 (1968)

10. Pucrto, A., Deutsch, J. A., Molina, F. \& Roll, P. Behav Biol. 18, 123-134 (1976).

11. Zahorik, D. M. in Learning Mechanisms in Food Selection ch. 8 (eds Barker, L. M., Best, M. R. \& Domjan, M.) 181-199 (Baylor Univ. Press, 1977)

2. Grote, F. W. Jr \& Brown, R. T. Behav. Res. Meth Insirum. 3, 311-312 (1971)

13. Dragoin, W. MoCleary, G. E. \& MoCleary, P. Behat Res. Meth. Instrum. 3, 309-310 (1971).

GibBS AND SMITH REPLY-We did not use a conditioned taste aversion paradigm in our study of the effect of bombesin (BBS) on feeding ${ }^{1}$ because this paradigm can no longer be considered a critical test of the presence or absence of malaise ${ }^{2}$. As a variety of agents (including isotonic saline and chlorpromazine, a drug with anti-nausea action) which serve as effective unconditioned stimuli for the formation of conditioned taste aversions ${ }^{3,4}$ do not produce sickness, the conditioned taste aversion test cannot be used as evidence of sickness. Conversely, as some rapidly acting rodenticides (including strychnine and cyanide) do not produce a conditioned taste aversion ${ }^{5}$, the failure to produce a taste aversion is not evidence that rats are not sick ${ }^{6}$. Thus, sickness is neither necessary nor sufficient for the formation of a conditioned taste aversion.

We rely instead on our demonstrations that BBS fails to affect the initial rate of feeding, fails to affect body temperature, fails to affect water ingestion in the range of doses that reduce food intake, and selectively affects feeding ${ }^{1}$. These results are good evidence that the effect we report on feeding is not due to sickness; none of these results would have been predicted if BBS were acting simply by producing illness. We have previously reported very similar observations ${ }^{7}$ as indications that the action of cholecystokinin (CCK) on food intake is not due to malaise, and it is important that these observations have proved to be excellent predictors of the results of human studies.

The satiety effect of CCK in humans has been dissociated from subjective reports of discomfort in three studies to date. The first report was that of Sturdevant and Goetz ${ }^{8}$, which Deutsch misquotes. These authors demonstrated that, while an intravenous (i.v.) injection of impure CCK at a high dose produced side effects, an i.v. injection of a lower dose significantly reduced food intake without causing any side effects or discomfort. This critical dissociation has now been reproduced twice in humans: pure or highly purified preparations of CCK reduce food intake ${ }^{9}$ and ratings of appetite $^{10}$ without producing reports of illness.

Deutsch concludes by assuming that CCK and BBS are producing malaise because the doses are unphysiological. We have previously shown that intraperitoneal injections of impure CCK as small as $2.5 \mathrm{Ivy}$ units $\mathrm{kg}^{-1}$ will reduce food intake in rats ${ }^{7}$ and that a slow i.v. infusion of a pure preparation of CCK as small as $30 \mathrm{ng} \mathrm{kg}^{-1}$ will reduce food intake in humans ${ }^{9}$. Both doses are within the ranges required to achieve the classic visceral effects of the hormone in each species, and are therefore likely to be physiological.

The evidence does not support Deutsch's dismissal of the actions of CCK and BBS on food intake. The possibility that these peptides have a role in satiety can be taken seriously.

\section{J. GibBS \\ G. P. SMIrH}

Department of Psychiatry,

Cornell University Medical College.

Bourne Laboratory;

The New York Hospital,

White Plains, New York 10605

1. Gibhs, J. et al. Nature 282, 208-210 (1979).

2. Smith, G. I $^{2}$ \& Gibbs, J. in Progress in Psyehobiellug and Physiological Psychology Vol. R (eds Sprague. J. M. E Epstcia. A. N.) 179242 (Acadomic. New York. 1979).

3. Bcrier B. D. J comp Physial Psychol 81, 3126 (1972)

4. Bcrger, B. D. J. comp. Physion Physiol. Behul: 6, 341-343 (1971)

5. Nachman, M. \& Hartley, P. L. J. comp. Phisiol. Psichot 89, 1010-1018 (1975).

6. Deutsch, J. A. \& Gonzalez. M. F. Behat. Biol. 24, 317 326 (1978).

7. Gibbs, J., Young, R. C. \& Smilh. (F. P. J. comnp. Phỵsiol Psychol. 84, 488-.495 (1973).

8. Sturdevant, R. A. L. \& Goct2, H. Nuture 261. 713715 (1976)

9. Kissiteff, H. R. Pi-Sunyer, F. X.. Thornton, J. \& Smith, Ci. P. C'lin. Kes. 27, 552A \{1979\}.

10. Slacher, (j.. Baucr. H. \& Steinringer, H. Physiel. Behar $23,325331(1979)$. 